\title{
Psychological Substantiation of Individual Typological Characteristics of Volleyball Players as Predictors of Success in Professional Activity
}

\author{
Novakovskiy S. \\ Institute of Physical Education, Sport and Youth Policy \\ First President of Russia B.N. Yeltsin Federal University \\ Yekaterinburg, Russia \\ s.v.novakovskiy@urfu.ru,
}

\author{
Shurmanov E. \\ Institute of Physical Education, Sport and Youth Policy \\ First President of Russia B.N. Yeltsin Federal University \\ Yekaterinburg, Russia \\ e.g.shurmanov@urfu.ru,
}

\author{
Kondratovitch S. \\ Institute of Physical Education, Sport and Youth Policy \\ First President of Russia B.N. Yeltsin Federal University \\ Yekaterinburg, Russia \\ s.v.kondratovich@urfu.ru
}

\begin{abstract}
The results of the female athletes' Central nervous system (CNS) and temperamental characteristics functional state study- students of the Olympic reserve school, professionally engaged in volleyball are presented. The analysis reflects the features that are typical for athletes of team sports. This is an attempt to consider the level of speed characteristics development through the prism of the identified individual characteristics of the NS. It was revealed that for a sample of female volleyball players important for success in the implemented activities are: the speed and stability of the visual-motor response, stability and concentration of attention, the type of nervous system. Temperamental features play an important role. At the same time, there is a direct relationship between the level of emotional instability and the speed of response $(r=0.68)$. Increasing the level of neuroticism leads to a decrease in stability and concentration $(r=0.59)$. The analysis of the results obtained in the course of the study made it possible to objectively assess the level of individual psychophysiological features of athletes to adjust the training process taking into account individual typological features.
\end{abstract}

Keywords - individual personality; volleyball athletes; motor activity.

\section{INTRODUCTION}

Sports activity places high demands on the preparedness of athletes in all sports. At the stage of maximum realization of individual opportunities, the personal qualities of the athlete acquire special importance. At the same time, it is important to assess the "sports talent" in the chosen sport, to determine the ways of developing the necessary abilities, the maximum potential of the athlete. Of particular importance is the psychological aspect that directly affects the success of the activity [2]. Skillful use of nervous system features and temperamental features of the athlete which are one of the most steady individual features of the person-a way to success of the athlete. The specificity of game sports, which include volleyball, makes high demands on the individual psychophysiological characteristics of athletes due to the fact that the psychomotor complexity of sports activity causes a specific set of indicators, manifested directly in the mental processes and personal characteristics. The reactivity of the athletes organism to training and competitive loads depends on many factors, and, first of all, on the mobility, strength and dynamism of nervous processes [1]. The introduction of modern psychodiagnostic tools-methodological tools with scientific justification - into the training process is designed to solve most issues related to modern tactical training of athletes, taking into account individual typological indicators due to innate inclinations.

\section{LITERATURE REVIEW}

The interrelation of different structures is given great importance and is considered by different domestic and foreign authors (E. p. Ilyin, 2005; G. D. Gorbunov, 2007; N. P. Filatova, 2011; W. P. Morgan, 1987; G. Tenenbaum, 2003, etc.) $[3,5,9,10,7,12]$. The priority side of these studies is the sport of higher achievements. Assessment of individual psychophysiological functions from the position of necessary 
The methods make it possible to determine the degree of potential opportunities development and their realization in the chosen sport at the stage of the athlete preparation is paid less attention, or this aspect is considered fragmentary. Given the above, it seems relevant to investigate the individual typological characteristics of athletes at the beginning of a sports career, which will allow in the future to properly carry out psychological and pedagogical impact in order to achieve the greatest success and efficiency in the activity.

The aim of the study is to assess the individual psychophysiological characteristics of female volleyball players aged 14-16 years to adjust the training program taking into account individual typological indicators in their relationship with typological features to achieve success in professional activities.

\section{RESEARCH METHODOLOGY}

Testing was carried out on the basis of the laboratory "Technologies of recovery and selection in sports" of the Ural Federal University during the recovery period (I period-may 2016; II period - April 2017).

The contingent of the study was a group of female athletespupils of the school of Olympic reserve of Yekaterinburg, engaged in volleyball. Age of the subjects aged 14-16 years. experience in volleyball is 4-6 years. Sports category -I-III. For the purpose of the received data reliability, in results of research indicators of the athletes who have taken part in all stages of testing $(\mathrm{n}=12)$ are presented.

The choice of diagnostic methods is determined by the specifics of sports activities of athletes:

for the purpose of determining the rate of simple visualmotor reaction - the technique is Simple visual-motor reaction (this method promptly) [6];

- to assess the complex response of nerve processes to the corresponding stimulus demonstrated, the technique of "choice Reaction" was applied, which is a kind of Complex visualmotor reaction (spmr) [11,14];

- determination of the motor apparatus speed characteristics, the pace and stability of the motor action was carried out using the Tapping test technique [3];

MP for the diagnosis of temperament type on the basis of determination of extra / introversion and neuroticism level MPI questionnaire by G. V. Eizenka [13].

During the testing of a simple visual-motor reaction, athletes with different time intervals were presented with light signals of the same color. When a signal appears, the subject should press the visual-motor analyzer button as soon as possible, trying to avoid premature pressing or skipping the signal.

When diagnosing a complex visual-motor reaction, the sequence of different colors signals is random. In response to the presentation of a signal of primary or secondary color, the subject had to press the corresponding button on the visualmotor analyzer as quickly as possible. attention stability due to the balance of nervous processes and to characterize the current functional state of the Central nervous system [4].

Express method Tapping test [3, 8] reflects the overall performance and strength of nerve processes. The test was carried out using two special devices: "pencil" and "rubber platform". The athlete was given the task to knock on the seconds. The results were processed by counting the number of movements performed in each of the five-second intervals of the test. On the basis of the results obtained, a graph characterizing the type of nervous system was constructed:

1. A strong nervous system is characterized by an increase in the speed of movement in the first 15 seconds by more than $10 \%$.

2. Medium strength nervous system: the speed of movement is maintained at the same level with fluctuations of $\pm 10 \%$ throughout the test.

3. The weakness of the nervous system is manifested by a descending type of line.

4. With a medium-weak nervous system, the maximum number of movements is fixed during the first five-second intervals, then the pace of movements falls.

5. Indicators of the medium-strong nervous system tend to decrease at the beginning of testing, then a short-term increase in the rate to the initial level $( \pm 10 \%)$ is recorded.

When diagnosing the type of temperament and personality traits, athletes were asked to answer test questions [13]. The results were evaluated according to the number of points scored on each scale. The relationship between the indicators of extra-or introversion (dependence of the characteristics of human activity on external / internal influences) and the level of neuroticism (emotional instability of the personality) of subjects with classical types of temperament is reflected in the so-called "circle" of Eysenck.

The list of analyzed parameters included: time this method promptly, varying in accordance with the state of the Central nervous system; Central delay - the difference between the value of time and this method promptly associated with the dissemination of information in the structures of NS; the level of functionality, which characterizes the ability of the CNS to retain the appropriate functional condition for accurate task execution. the degree of concentration and stability of attention; the rate of strokes to determine the parameters of the nervous system properties; the degree of emotional instability and extra - or introversion.

To identify the individual characteristics of athletes were used the capabilities of the computer complex " NS-Psychotest "(firm "Neurosoft" Ivanovo, Russia). Data analysis was carried out using the statistical software package "SPSS Statistica, v 23.0". Comparison of empirical data in the dynamics of the studied period was carried out using the nonparametric Kruskal-Wallis criterion; identification of the relationship between the studied indicators - using the RPearson correlation analysis method. platform with the maximum possible frequency for 30 


\section{RESULTS}

The analysis of simple visual-motor reaction indicators aimed at identifying the speed of response to the appearance of an object in the field of vision and complex visual-motor reaction characterizing the ability to quickly move from one action to another in the process of intensive activity showed the dynamics in the manifestation of the studied indicators in accordance with the selected time parameters. The parameters of the test for determining the simple and complex visualmotor reaction of athletes are presented in table I.

TABLE I. PERFORMANCE CHARACTERISTICS OF THE ATHLETES RESPONSE RATE

\begin{tabular}{|c|c|c|}
\hline \multirow[t]{2}{*}{ Characteristic } & \multicolumn{2}{|c|}{ Period } \\
\hline & $\begin{array}{c}\mathrm{I} \\
\mathrm{M} \pm \mathrm{SD} \\
(\min -\mathrm{max})\end{array}$ & $\begin{array}{c}\mathrm{II} \\
\mathrm{M} \pm \mathrm{SD} \\
(\min -\mathrm{max})\end{array}$ \\
\hline $\begin{array}{l}\text { Time this method } \\
\text { promptly, MS }\end{array}$ & $\begin{array}{c}219,7 \pm 39,7 \\
(173,45-243,17)\end{array}$ & $\begin{array}{c}195,72 \pm 14,2 \\
(158,18-214,25)\end{array}$ \\
\hline Time spmr, MS & $\begin{array}{c}320,87 \pm 29,2 \\
(286,22-332,4)\end{array}$ & $\begin{array}{c}316,03 \pm 21,8 \\
(250,35-320,8)\end{array}$ \\
\hline VCZ, MS & $\begin{array}{c}101,17 \pm 21,1 \\
(69,43-118,4)\end{array}$ & $\begin{array}{l}120,3 \pm 24,5 \\
(68,7-143,5)\end{array}$ \\
\hline $\begin{array}{l}\text { The stability of the } \\
\text { reaction response, MS }\end{array}$ & $\begin{array}{c}134,1 \pm 13,3 \\
(46,72-159,8)\end{array}$ & $\begin{array}{l}39,6 \pm 13,5 \\
(40,1-69,6)\end{array}$ \\
\hline $\begin{array}{c}\text { Concentration, attention. } \\
\text { ed. }\end{array}$ & $\begin{array}{c}0,12 \pm 0,07 \\
(0,00-0,20)\end{array}$ & $\begin{array}{c}0,06 \pm 0,03 \\
(0,00-0,17)\end{array}$ \\
\hline $\begin{array}{l}\text { UVB this method } \\
\text { promptly, C2 }\end{array}$ & $\begin{array}{c}4,2 \pm 0,63 \\
(4,19-4,97)\end{array}$ & $\begin{array}{c}3,9 \pm 0,54 \\
(3,31-4,2)\end{array}$ \\
\hline
\end{tabular}

As follows from table 1, the average reaction time of female volleyball players for the studied periods of the annual cycle was 195-219. 7 MS., which indicates an improvement in the speed of visual-motor reaction of the subjects. Analysis of the results of a simple visual-motor reaction for the research periods showed. The majority of subjects $(66 \%)$ showed stable results at the end of the two stages of the study, slightly varying their performance. It should be noted that the values of the four subjects during all stages of the study showed an unstable nature of the reaction rate, that is, the result had significant differences.

Indicators of complex response time (320.87-316.03 MS) reflect the average speed of information processing and are inherent in the majority of team members $(55 \%)$. It can be assumed that in the process of systematic sports games, athletes develop the ability to adequately choose a solution from several alternatives.

The parameters of the central delay time are the most variable under the influence of loads. Slowness in the actions of the player is due to the lengthening of the information period processing in the structures of the NS. The results obtained have no significant differences and correspond to the average level of reaction speed.

The index of response stability and balance of nervous processes showed that the most stable results were recorded following the results of the research II stage and, on average, the standard deviation was 39.6 MS with a span of 35-134 MS. This is explained by the fact that players can not always cope with their emotions, and the predominance of excitement emotions affects the stability of the task.

When determining the degree of attention stability, the ratio of the errors number missed and premature reactions to the presentation of the stimulus is considered. The coefficient of accuracy was reduced from $0.12 \%$. units for the first period up to $0.060 \%$ after the final control, indicating that the degree of attention stability also has a positive trend. It should be noted that the rate of sensorimotor reaction is influenced by the body functional state. As can be seen from the data presented in table I, this indicator is at an average level and also tends to stabilize.

In sports activities foresight is a prerequisite for the success of the athlete. The study of response strategies showed a significant predominance of the number of accurate reactions over the number of errors (75 and $16 \%$, respectively) in response to the presentation of the signal provoking the excitation processes (table 2). The results indicate success in activities under difficult conditions.

TABLE II. INDICATORS OF NERVOUS PROCESSES MOBILITY CHARACTERISTICS

\begin{tabular}{|c|c|c|}
\hline Characteristic & $\begin{array}{c}\text { Group performance, } \\
\%\end{array}$ & $\begin{array}{c}\text { Number of persons } \\
(\mathrm{n}=12)\end{array}$ \\
\hline $\begin{array}{c}\text { Index of accurate } \\
\text { reactions }\end{array}$ & $75 \%$ & 9 чел \\
\hline False reaction rate & $16 \%$ & 2 чел. \\
\hline Lead rate & $33 \%$ & 4 чел. \\
\hline Delayed reaction rate & $8 \%$ & 1 чел. \\
\hline
\end{tabular}

Figures in table II indicate the formation of the nervous processes management skills most of the subjects, which is reflected in the number of accurate responses and a low incidence of false and delayed reactions in the group of subjects.

To assess the strength of nerve processes, the following parameters of the tapping test were studied: the average frequency of strokes $(\mathrm{Hz})$; the initial and peak frequency of strokes $(\mathrm{Hz})$; the strength of nerve processes and their changes in the annual training cycle. The results of the experimental data processing allowed to evaluate the speed characteristics of the motor apparatus and determine the type of subjects with different CNS strength and conditionally divide the group of subjects into types in accordance with the criteria of E.P. Ilyin.

Determination of the nervous system type showed that the majority of test subjects $(76.93 \%)$ have a medium-sized type of nervous system. Athletes of this type have the ability to react quickly to an emergency situation. The revealed feature is explained by the fact that volleyball is a sport in which athletes constantly train the switchability of the CNS processes when performing tasks. Weak type of NS is inherent in $15.38 \%$ of subjects. Psychologically, such athletes are impressionable, acute reaction to stress, very susceptible to praise and rewards. It should be noted that $7.69 \%$ of the subjects have a strong type of NS, that is, the ability to tolerate strong excitation or inhibition. 
NS and stability of reaction does not differ reliability, nevertheless, the weak correlation indicating that the most effective activity is observed at the optimum functional state corresponding not too high or low level of activation of NS is traced.

TABLE III. CORRELATION MATRIX OF THE NERVOUS SYSTEM INDIVIDUAL TYPOLOGICAL FEATURES INDICATORS AND THE LEVEL OF NEUROTICISM OF ATHLETES activity in conditions of long-term intensive load, as indicated by the average value of strokes for 5 seconds $(7,2 \mathrm{~Hz} ;>30$ beats), and confirms the ability of the subjects to withstand intense load.

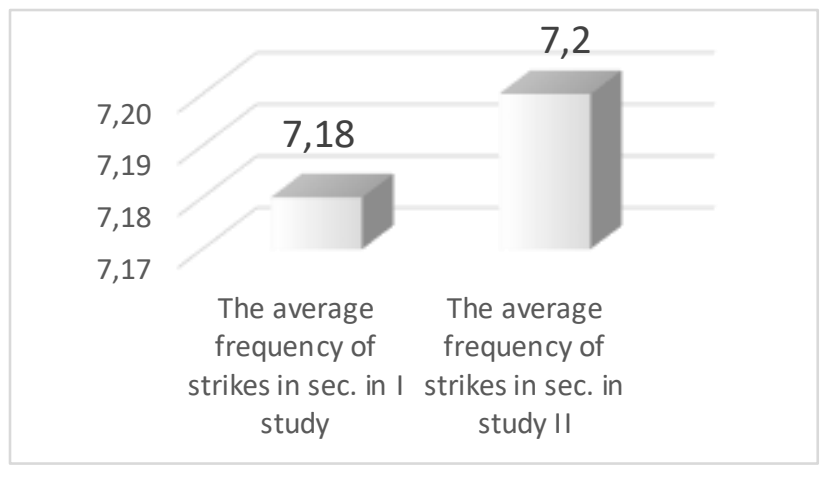

Fig.1. Indicators of the subjects motor action, $\mathrm{Hz}$

The importance of the temperament study in its connection with the types of the nervous system is quite undoubted in matters sports training construction methods. The results of determining the psychological characteristics of the individual, taking into account the degree of individual psychological properties severity, and the degree of the data reliability indicate that in the group of subjects $42 \%$ are athletes of a balanced type of temperamental characteristics (closer to sanguine), $23 \%$ - choleric-sanguine type, $28 \%$ have a pronounced choleric type and only $7 \%$ (1 subject) melancholic type of temperament. The conducted testing of temperamental features in accordance with G. V.'s methodology. Eisenke allows the determination of such parameters as the level of anxiety (neuroticism) and personality orientation (extra / introversion). The results showed that the group of subjects is dominated by the characteristics of extroverted type and increased levels of neuroticism. Psychologically, such athletes are sensitive, impressionable, acute reaction to stress, very susceptible to praise and rewards. It can also be assumed that due to the age of the subjects (14-16 years), some of the subjects have increased anxiety and questionable reliability of answers as an indicator of social attractiveness (seem better than you really are).

To determine the possible relationship between the studied indicators, a correlation analysis was carried out (table 3).

As follows from the correlation calculations, there is a direct relationship between the level of emotional instability of the individual and the speed of response $(\mathrm{r}=0.62)$. An increase in neuroticism leads to a decrease in the stability of attention $(\mathrm{r}=0.56)$. The selected criterion does not have a significant impact on the ability to perform intensive activities. And though the fact of a psychoemotional state change in its connection with indicators of level of functional capabilities of

\begin{tabular}{|l|c|c|}
\hline \multicolumn{1}{|c|}{ The parameters } & $\begin{array}{c}\text { Level of } \\
\text { neuroticism }\end{array}$ & $\begin{array}{c}\text { Level of } \\
\text { significance (r) }\end{array}$ \\
\hline Simple reaction rate, MS & $0,68^{*}$ & $<0,05$ \\
\hline $\begin{array}{l}\text { The stability of the reaction } \\
\text { response, MS }\end{array}$ & 0,45 & $>0,05$ \\
\hline $\begin{array}{l}\text { Stability and concentration of } \\
\text { attention, ed. }\end{array}$ & $0,59^{*}$ & $<0,05$ \\
\hline $\begin{array}{l}\text { Level of functionality, usl. ed. } \\
\text { The average frequency of } \\
\text { strokes (Hz) }\end{array}$ & 0,32 & $>0,05$ \\
\hline $\begin{array}{l}\text { The ratio of the nervous } \\
\text { system strength }\end{array}$ & 0,39 & $>0,05$ \\
\hline $\begin{array}{l}\text { Note: correlation coefficients (g) with the level of statistical significance are indicated: * }-\mathrm{p}<0,05: * *- \\
\mathrm{p}<0,001\end{array}$ & 0,27 & $>0,05$ \\
\hline
\end{tabular}

\section{CONCLUSION}

1. The conducted testing included simple, but informative methods, which allowed to determine the most complete individual typological characteristics of volleyball players.

2. Monitoring of the subjects psychophysiological features showed that the athletes demonstrate a high reaction rate (195-219, $7 \mathrm{MS})$; indicators of complex response time (320.87-316.03 MS) reflect the average speed of information processing and are inherent in the majority of team members (55\%). $76.93 \%$ of athletes are capable of high-intensity activity, $7.69 \%$ of subjects-to the regulation of nervous processes with strong excitation or inhibition.

3. The proposed monitoring of the mental processes sphere indicators and conditions of athletes, in accordance with the degree of individual psychological properties expression, makes it possible:

a) take into account the level of individual typological features development in the targeted training of athletes;

b) effectively to carry out psychological and pedagogical influences taking into account the revealed individuallytypological features of athletes;

C) timely correction of the training process, which will undoubtedly contribute to the optimal development of players to achieve success in professional activities. 
[9] Tenenbaum, G., Jones, C.M., Kitsantas, A. et al.. Failure adaptation: an investigation of the stress response process in sport. International Journal of Sport Psychology. 2003, 34(1), pp. 27-62

[1] Alekseeva E.E. Psychophysiological Diagnostics of Typological Human Features. Proceedings of the Scientific Conference "Ananiev Readings" Modern Applied Directions and Problems of Psychology", October 19-21, 2010. S.-Petersburg, Russia. 2010, pp. 7-8.

[2] Gould D, Dieffenbach K., Moffett A. Psychologian characteristics and their development in Olimpic champions. J. Appl. Sport psychol.14. 2002, pp. 172-204.

[3] Ilyin, E.P. Psychophysiology of human states. St. Petersburg: Peter. 2005, pp. 415

[4] Loskutova, TD Response Time as a Psychophysiological Method for Evaluation of the Functional Condition of the CNS. Neurophysiological Studies in the Examination of Work Capacity. L .: Medicine. 1978, pp. 165 .

[5] Morgan, W.P., Brown, D.R., Raglin, J.S. et al. Physiological monitoring of overtraining and staleness. British Journal of Sports Medicine. 1987, 21, pp. 107-140

[6] Neurophysiological studies in the examination of work capacity / Ed. A.M. Zimkina, V.I. Klimovoi-Cherkasova. L : Medicine. 1978, pp. 125.

[7] Park, I.S. Basketball training increases striatum volume. Hum Mov Sci. 2011 Feb, 30(1), pp.56-62. Epub 2010 Oct 27.

[8] Runenko S.D. The study and evaluation of the functional condition of athletes: Textbook. M .: Profi., 2010, pp. 172

[10] Zakharova A., Mekhdieva K. and Kondratovitch S. Physical and Psychophysiological Profiles of Sub-elite Basketball Players - Novel Approach to Complex Testing. Vol.: icSPORTS. 2017, pp. 132-139. doi 10.5220/0006585401320139

[11] Zimkina, A.M. Neurophysiological studies in the examination of work capacity. L: Medicine. 1978, pp. 221

[12] Ziv, G., Libor, R. Physical Attributes, Physiological Characteristics, On-court Performances and Nutritional Strategies of Female and Male Basketball players. Sports Medicine. 2009, pp. 547-568.

[13] Vyatkin, B. A. the Role of temperament in sports. M.: "Physical Culture and sport". 1978, pp. 134

[14] Aleksandr S. Kuznetsov, Evgeniya N. Usmanova, Oksana V. Kolomytseva. Athletes' psychological-physiological indices study in different specializations at the stages of sports career crises overcoming. 2019, 14(2), pp. 89-96. DOI: 10.14526/2070-4798-201914-2-98-96.

[15] Workshop on General, experimental and applied psychology: textbook. St. Petersburg: Peter. 2000, pp. 254. 